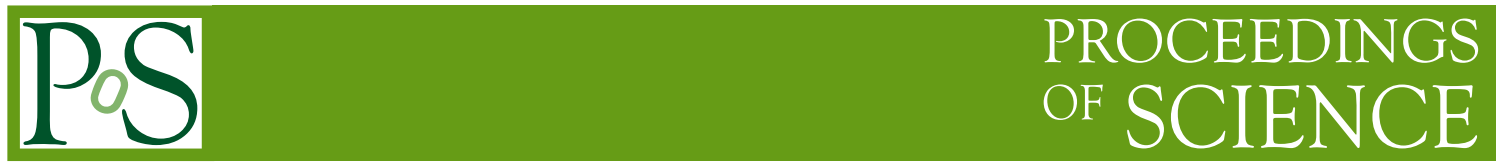

\title{
Recent Advances in Cosmological Hydrogen Reionization
}

\author{
Kristian Finlator* ${ }^{\dagger}$ \\ UC Santa Barbara \\ E-mail: finlatorephysics.ucsb.edu
}

\begin{abstract}
I discuss recent advances in the study of hydrogen reionization, focusing on progress that was achieved during the years 2010-2011. First, I discuss recent measurements of the progress of reionization. Next, I discuss recent observational constraints on the nature and abundance of the dominant ionizing sources. Finally, I discuss recent progress in modeling reionization. This review is written for an audience of astronomers who do not specialize in the high-redshift Universe.
\end{abstract}

Frank N. Bash Symposium New Horizons In Astronomy,

October 9-11, 2011

Austin Texas

\section{${ }^{*}$ Speaker.}

$\dagger$ I am grateful to the University of Texas Department of Astronomy Board of Visitors for supporting the 2011 Frank N. Bash Symposium. I also thank NASA for support through Hubble Fellowship grant HF-51254.01 awarded by the Space Telescope Science Institute, which is operated by the Association of Universities for Research in Astronomy, Inc., for NASA, under contract NAS 5-26555. 


\section{Introduction}

The Reionization Epoch consists broadly of the time interval between the moment when photons and matter decoupled, at a redshift $z$ of 1088 [73], and the moment when enough ionizing sources had formed to ionize the hydrogen and helium in the intergalactic medium (IGM), or $z \sim 3$ [69]. This was the most active period in the Universe's history: Galaxies were forming stars up to ten times as rapidly as they are today even as they expelled comparable amounts of gas back into their environments. The supermassive black holes that are largely dormant today were each swallowing a dwarf galaxy's worth of gas over just a few billion years and glowing brightly enough to heat the IGM. Reionization converted the IGM into a plasma, suppressed the growth of dwarf galaxies, and altered the conditions under which subsequent generations of galaxies grew. Its pivotal position at the outset of the story of structure formation rendered it one of the central science goals in the 2010 Decadal Survey [1].

The Reionization Epoch is generally divided in two: During the hydrogen reionization epoch, hydrogen was ionized and helium was singly ionized. During the helium reionization epoch, helium was further ionized from He II to He III. Two considerations justify this division. First, most of the photons that brought about hydrogen reionization were not energetic enough to remove the second electron from a helium atom. At the same time, the cross section for hydrogen atoms to absorb photons that are energetic enough to ionize a HeII atom is small. Hence the dominant photons were different. Second, hydrogen-ionizing photons were probably generated by massive stars in young galaxies while He II-ionizing photons were predominantly generated by quasars. Quasars did not grow abundant enough to participate in reionization until hydrogen reionization was largely complete [67]. Hence the sources were also different.

This review focuses on hydrogen reionization (from now on, "reionization"). I shall discuss insights that have been won during 2010-2011, with reference to earlier results whenever necessary for context. This field is routinely reviewed. For a more technical introduction on early structure formation, see the classic discussions by Barkana and Loeb [3, 45]. For overviews of the first galaxies and their role in reionization, see [14] and [11].

I divide my discussion into three parts. In Section 2, I discuss progress in measuring the history of reionization. When did reionization begin ? How long did it take? In Section 3, I discuss progress in identifying and understanding the ionizing sources that drove reionization. What were they? How many were there? Did the progress of reionization leave any observable signatures on their properties? In Section 4, I discuss progress in modeling reionization.

\section{Measuring the Progress of Reionization}

It is currently believed that reionization was driven by ionizing photons from dwarf galaxies. If true, then its history can be divided into three stages [29]. During the first stage, galaxies create enough ionizing photons to reionize their immediate surroundings, but the ionized regions remain small compared to the typical distance between galaxies. During the second stage, ionized regions begin overlapping with each other and the typical distance traveled by ionizing photons grows rapidly. During the final stage, the Universe is largely ionized and its residual opacity is dominated 
by slowly-shrinking "islands" of moderately overdense gas. In this Section, I discuss efforts to reconstruct when the Universe went through these stages.

\subsection{Lyman- $\alpha$ absorption}

The classic indication that reionization has already completed is the absence of a "GunnPeterson trough" from the spectra of high-redshift quasars. Intuitively, the cross-section for neutral hydrogen atoms to absorb photons that boost their electrons from the ground state $(n=1)$ to the first excited state $(n=2)$ is very large. Any photon that is in resonance with this transition (Ly $\alpha)$ while traversing a region with neutral hydrogen will probably be absorbed. [33] showed that, if the volume-averaged neutral hydrogen fraction $x_{\mathrm{HI}}$ is $\sim 1$, then the optical depth to scattering is $\sim 10^{4}$. Put differently, as UV photons redshift through resonance with Ly $\alpha$, they will on average be scattered unless fewer than 1 in $10^{4}$ hydrogen atoms is neutral. In the post-reionization Universe, this phenomenon creates a "forest" of absorption lines, where the amount of absorption at a restframe wavelength in the range 912-1216 $\AA$ traces the amount of neutral gas in the Universe at the moment when those photons redshifted through resonance with $\operatorname{Ly} \alpha$. In a reasonably neutral Universe, the forest converts into complete absorption, and is called the Gunn-Peterson trough.

The first reported Gunn-Peterson trough was identified in the spectrum of a quasar at $z=$ 6.28 [5], leading to speculation that reionization may have ended around this redshift. In practice, however, the detection of a Gunn-Peterson trough only indicates that $x_{\mathrm{HI}}>10^{-3}$ [18], which is still highly ionized. [19] followed up on this by applying several complementary analyses to 19 quasars, tracing the evolution of $x_{\mathrm{HI}}$ from $5 \rightarrow 6$. They found that $x_{\mathrm{HI}}$ evolves rapidly during this interval and inferred that the IGM is mostly neutral at $z \geq 6$. In detail, their analyses all assumed a spatially homogeneous ionizing background, which is clearly an approximation near the end of reionization. Relaxing this assumption leads to the more conservative limit $x_{\mathrm{HI}}(z=6.1)<0.8$ [47].

A complementary constraint on $x_{\mathrm{HI}}$ comes from modeling the "proximity zones" of highredshift quasars. A proximity zone is a region centered on a quasar that is more ionized than the ambient IGM owing to the quasar's extra ionizing photons. Its size, which can be measured and is typically a few Megaparsecs, is sensitive to (1) the quasar's ionizing luminosity; (2) the neutral hydrogen fraction before the quasar "turned on"; and (3) the amount of time that the quasar has been active. The quasar's ionizing luminosity can be constrained observationally, so if the duration of its active phase can be derived through other means then $x_{\mathrm{HI}}$ can be inferred using a straightforward Strömgren Sphere calculation [67].

Recently, [52] and [7] applied this approach to a newly-discovered quasar at $z=7.085$ called ULAS J1120+0641. Its proximity zone is unusually small in comparison to other high-redshift quasars, and it is the smallest when its radius is scaled to the quasar's luminosity. This is consistent with the IGM being more neutral than at lower redshifts. Of course, it is also possible that the proximity zone is small because the quasar just "turned on". Fortunately, the UKIDSS will soon yield more quasars at similar redshifts, enabling independent measurements of $x_{\mathrm{HI}}$ [36].

It is also possible to infer $x_{\mathrm{HI}}$ from stronger $\operatorname{Ly} \alpha$ absorbers. If a region's neutral hydrogen column density exceeds $10^{20.3} \mathrm{~cm}^{-2}$ (and it will if the IGM is neutral), then Ly $\alpha$ absorption enters the damped portion of the curve of growth. In this case, the red damping wing's shape constrains $x_{\mathrm{HI}}$. [75] used the absence of a damping wing in the afterglow spectrum of a gamma-ray burst to infer that $x_{\mathrm{HI}}(z=6.3)<0.17$. By contrast, [7] find evidence for this feature in the spectrum of 
ULAS J1120+0641, and they infer from it that $x_{\mathrm{HI}}(z=7.085)>0.1$. Of course, it is also possible that the surrounding IGM is highly ionized but a neutral parcel of gas (such as a satellite galaxy) happens to sit just in front of the quasar. This possibility again indicates the need for a larger sample of reionization-epoch quasars.

\subsection{Redshifted hyperfine emission from neutral hydrogen}

A hydrogen atom has slightly lower energy when the spins of its proton and electron are antiparallel than when they are parallel, and it can transition between these energy states by emitting or absorbing a photon of wavelength $21.1 \mathrm{~cm}$. Early in the reionization epoch, when the IGM had been warmed by quasars and stars but not yet ionized, the entire Universe was glowing brightly in this transition. Many of those photons were never re-absorbed, leading to a background that should be visible today at a frequency of $1420.4 \mathrm{Mhz} /(1+z) \approx 140 \mathrm{Mhz}$. In fact, it should be possible to image the sky at a range of frequencies around $140 \mathrm{Mhz}$ and document the progress of reionization [76] either by directly imaging brighter neutral and fainter ionized regions or through statistical approaches. In practice, the observation is challenging because the expected flux is five orders of magnitude weaker than foreground sources. Nonetheless, many low frequency radio experiments are under development that will use novel calibration techniques to isolate this signal and map out the history of reionization [51].

Recently, [10] used the simpler approach of measuring the all-sky spectrum of redshifted $21 \mathrm{~cm}$ emission. They inferred that, if reionization occured between $z=13$ and $z=6$, then its duration was longer than $\Delta z>0.06$ with $95 \%$ confidence. Models generally predict that its duration was significantly longer than this, so it is not yet a strong constraint. Nonetheless, it is the first result from what promises to be a rich field over the coming decades.

\subsection{The Cosmic Microwave Background}

Another approach to constraining the timing of reionization involves measuring the impact that Thomson scattering by free electrons had on the anisotropies in the cosmic microwave background (CMB). This is quantified by $\tau_{\mathrm{es}}$, which can be thought of as the probability that a given CMB photon scattered at some redshift below roughly 30 . Measurements by the WMAP satellite indicate that $\tau_{\mathrm{es}}=0.088 \pm 0.015$ [44]. Of this, 0.050 originates from the portion of the photon's path between $z=7$ and $z=0$. The remaining 0.035 is an integral constraint on the history of reionization. It could indicate that reionization occurred over a short interval (but longer than $\Delta z=0.06$ !) around $z=10.6$, but it is equally consistent with some scenarios in which reionization began at $z=14$ and completed around $z=6$ (see Figure 4 of [85]).

Additional constraints come from much smaller-scale fluctuations in the CMB. The idea is that $\mathrm{CMB}$ photons can gain energy by inverse-Compton scattering off of energetic electrons. If the scattering electrons reside within a galaxy cluster, then they create "hot spots" in CMB maps; this is called the thermal Sunyaev-Zel'dovich (tSZ) effect. If the scattering electrons exhibit bulk motions, then it is called the kinetic Sunyaev-Zel'dovich (kSZ) effect. Some kSZ scattering occurs after reionization, but an additional contribution is expected from ionized patches at $z>6$. The kSZ signature constrains the duration of reionization because the distribution of ionized and neutral patches that a line of sight passes through depends on how long reionization lasted. Since 
2007, the South Pole Telescope (SPT) and the Atacama Cosmology Telescope (ACT) have been measuring small-scale fluctuations in the CMB that are sensitive to this effect. [85] recently combined measurements from the SPT and WMAP, finding with $95 \%$ confidence that reionization had a duration of less than $\Delta z=7.9$ and ended before $z=5.8$. Their inferences will improve with more data and a better understanding of dusty star-forming galaxies at lower redshifts.

\subsection{Signatures of Reionization on Galaxies}

Star-forming galaxies irradiate their interstellar media (ISMs) with ionizing photons from massive stars. Two thirds of the resulting hydrogen recombinations produce Ly $\alpha$ photons. Now, Ly $\alpha$ is the lowest-energy transition to the ground state of hydrogen. This means that a Ly $\alpha$ photon in a neutral region will be repeatedly absorbed and re-emitted, performing a random walk until it either escapes or is destroyed. The photon is "destroyed" if an absorbing atom decays through two-photon emission. It can also be destroyed through dust absorption or OIII Bowen fluorescence. If the IGM is fully ionized, then any photons that escape their galaxy's ISM will no longer scatter. This is the case at $z<6$, where many galaxies show strong $\operatorname{Ly} \alpha$ emission lines [54]. If a galaxy emits Ly $\alpha$ photons into an IGM that is not fully ionized, however, then its $\operatorname{Ly} \alpha$ photons will be scattered into a low surface-brightness halo. This has several measureable consequences.

The luminosity function of $\operatorname{Ly} \alpha$ emitters should evolve. Galaxies that are selected to have bright Ly $\alpha$ emission lines are called $\operatorname{Ly} \alpha$ emitters (LAEs). Models indicate that, at epochs during which the IGM is more than $50 \%$ ionized, the observed abundance of LAEs should drop precipitously owing to IGM scattering [26, 49]. Recently, three studies reported that the luminosity function (LF) of LAEs decreases from $z=5.7$ to $z=6.5[37,57,41]$. This evolution could owe to a partially-neutral IGM at $z=6.5$, but it could also owe to an overall decline in the abundance of galaxies. [41] therefore verified that the distribution of UV-continuum fluxes from their LAEs at $z=5.7$ and $z=6.5$ are similar. This conflicts with the view that the $z=6.5$ sample is fainter in Ly $\alpha$ owing to lower star formation rates and suggests a partially-neutral IGM. If so, [41] inferred that $x_{\mathrm{HI}}$ increases from 0 to $0.3-0.4$ from $z=5.7 \rightarrow 6.5$. However, the amount by which the LAE LF evolves differs between the different studies, leading to uncertainty in the strength of the constraint on reionization. Larger samples will eventually settle this question.

The $\operatorname{Ly} \alpha$ escape fraction should evolve. A complementary way to identify IGM attenuation on $\operatorname{Ly} \alpha$ emission lines is to identify galaxies at a range of redshifts using a selection technique that does not involve $\operatorname{Ly} \alpha$, then explore the evolution in their Ly $\alpha$ properties. Samples that push into the reionization epoch should have suppressed $\operatorname{Ly} \alpha$ emission for the same reasons as before. Galaxies that are identified via the Lyman-dropout technique can be used in this way [68].

Over the past two years, evidence has accumulated that the equivalent width of $\operatorname{Ly} \alpha$ emission from such samples grows with increasing redshift to $z=6$ and then drops to higher redshifts [57, $56,60,66,74]$. This evolution is often referred to as an evolving "escape fraction" of Ly $\alpha$, but its physical interpretation remains controversial. [56] present evidence that the Ly $\alpha$ escape fraction drops for faint objects but not for bright ones and interpret this as a constraint on the topology of reionization, or the order in which regions of different densities reionized. This conflicts with the expectation that reionization will suppress the abundance of bright Ly $\alpha$ sources by the same factor as faint ones $[26,49]$. Therefore, differential evolution in the Ly $\alpha$ escape fraction could also be a signature of differential evolution in galaxies' ISMs. If, for example, the gas fraction evolves 
rapidly for low-mass galaxies, then they could simply destroy their Ly $\alpha$ photons more effectively at higher redshift. Settling this question will require improved constraints on bright and faint galaxies.

The clustering of LAEs should increase. Galaxies are not distributed uniformly in space, and the degree to which they cluster constrains the dominant growth processes. Conceptually, observers quantify clustering through the enhanced probability that a galaxy has a neighbor at a given distance over what would be expected if galaxies were distributed uniformly. If galaxies produced the photons that drove cosmological reionization, then their clustering influences the history of reionization. In particular, by the time that $x_{\mathrm{HI}}$ has dropped to $\sim 50 \%$, the average ionized gas parcel sits within an ionized region that is of order 10 comoving Megaparsecs in radius and whose ionization is maintained by many hundreds of galaxies working together. A Ly $\alpha$ photon emitted within such a region redshifts out of resonance with Ly $\alpha$ after traveling $\sim 1 \mathrm{Mpc}$ [12]. By the time it reaches the neutral IGM, it will no longer be able to scatter. The implication is that LAEs ought to exhibit increased clustering as observations push into the reionization epoch because Ly $\alpha$ photons from galaxies in crowded neighborhoods will travel farther before reaching the neutral IGM. [57] searched for this signal and found that LAEs' clustering properties at $z=6.6$ were indistinguishable from samples at lower redshifts, suggesting that the IGM was already more than $50 \%$ ionized by $z=6.6$.

The mean shape of the Ly $\alpha$ line profile should evolve. As $x_{\mathrm{HI}}$ increases, the tendency for the IGM to scatter the Ly $\alpha$ line should evolve more strongly on the blue side of the line than on the red side. Consequently, the typical profile of the $\operatorname{Ly} \alpha$ line could evolve in redshift. [57] compared the stacked line profiles of LAEs at $z=5.7$ and $z=6.6$, finding at most $1 \sigma$ evidence for evolving line profiles. This is inconsistent with some models in which $x_{\mathrm{HI}}$ increases from 0 to $>0.5$ during this redshift interval.

In summary, some of the expected signatures of reionization on the Ly $\alpha$ properties of galaxies have been detected while others have not. Unfortunately, the interpretation of these observations remains unclear. A major source of uncertainty involves the role that bulk gas motions could play in modulating $\operatorname{Ly} \alpha$ line profiles. For example, gas outflows can endow Ly $\alpha$ photons with a net redshift, making it easier for them to avoid scattering off of the IGM once they emerge from the galaxy. [15] showed that this effect can allow more than half of all Ly $\alpha$ photons to avoid scattering even if $x_{\mathrm{HI}}=0.6$. More generally, a consensus as to the physical interpretation for Ly $\alpha$ line profiles does not yet exist owing to degeneracies between the processes that could affect them. For this reason, observations of $\operatorname{Ly} \alpha$ emission cannot by themselves constrain the history of reionization even though they yield a variety of useful and complementary clues.

\section{Measuring the Sources of Reionization}

Reionization clearly happened. Hence while some investigators reconstruct its timing, others ask which ionizing sources could have been responsible. The sources that can be constrained observationally are quasars and galaxies; I shall touch on more speculative sources in Section 4.

Quasars did not dominate reionization. In particular, by measuring their abundance, estimating their total ionizing luminosity, and comparing it to the IGM recombination rate, many studies have shown that quasars could not have contributed more than half of all ionizing photons at $z>$ $4[67,28]$ and only $1-5 \%$ at $z=6$ [79]. This leaves galaxies, which in turn raises two questions: 
(1) How many galaxies were there as a function of luminosity and redshift? and (2) How many ionizing photons did they emit into the IGM?

Counting Galaxies By counting galaxies and making simple assumptions regarding their ionizing luminositites, it is possible to show that the observed galaxies could not have kept the Universe ionized at $z=6$. What about fainter galaxies? The extrapolated abundance of faint galaxies is quantified by the slope of the power law that fits the LF's faint end. Galaxies may drive reionization as long as this slope, $\alpha$, is steeper (more negative) than -1.6 [82, 62]. A number of groups have recently used extraordinarily deep images taken by the Wide Field Camera 3 (WFC3) on board the Hubble Space Telescope (HST) to constrain the abundance of Lyman dropout galaxies out to $z=10[21,8,17,31,32,48,55]$. A consensus is emerging that $\alpha$ is between -1.8 and -2 for $z \geq 6$. In a complementary study, [35] measured the faint-end slope of the LAE LF at $z=5.7$, finding $\alpha=-1.7$. Both results are consistent with the view that faint galaxies drove reionization.

A complementary way to constrain the activity in galaxies involves counting gamma-ray bursts (GRBs). "Long" gamma-ray bursts are associated with core-collapse supernovae, hence their abundance tracks the total star formation rate density. This inference is subject to its own systematic uncertainties, but unlike galaxy-counting studies it does not require a correction for the contribution from faint galaxies. Recent work indicates that the GRB abundance implies enough star formation in galaxies to complete reionization by $z \approx 8[42,80,63]$.

Inferring Their Ionizing Luminosities How many ionizing photons did each galaxy emit into the IGM? This depends on the metallicity and age of its stars as well as the amount of dust in its ISM. Each of these properties also impacts the ultraviolet (UV) continuum ( $\lambda \approx 912-3000 \AA)$ of a galaxy's spectrum. Broadly, galaxies with young stars, low metallicities, and low amounts of dust have bluer UV continua and higher ionizing luminosities. Hence constraining the UV continua of reionization-epoch galaxies is a way to learn about their ionizing luminosities.

Recent studies have used WFC3 observations to show that fainter reionization-epoch galaxies are bluer $[9,22]$. They interpret this trend as evidence that fainter galaxies have less dusty ISMs. If so, then dust was nearly absent from the faint galaxies that probably dominated reionization. The UV continuum luminosity is then a more direct tracer of the ionizing luminosity than at lower redshifts, where the UV continuum is invariably modulated by dust extinction. Moreover, a larger fraction of ionizing photons probably escape into the IGM. Both of these studies also find that the UV continuum slopes lie within the range expected for moderately metal-enriched stellar populations. In other words, observations do not yet provide evidence for the long-sought zero-metallicity stars, which are expected to show very blue UV continua [64].

What fraction of ionizing photons escape into the IGM and participate in reionization? Clearly, this question is impossible to answer directly because ionizing photons emitted by reionizationepoch galaxies were absorbed by the IGM. However, it is possible to constrain the ionizing escape fraction, $f_{\text {esc }}$, at lower redshifts. [71] and [53] recently used direct detections of ioinizing photons from galaxies at $z \sim 3$ to show that the ratio of escaping ionizing continuum flux $F_{\text {LyC }}$ to 1500 $\AA$ flux $F_{1500}$ increases both with increasing redshift and decreasing luminosity. This suggests that $f_{\text {esc }}$ increases to high redshift and faint luminosity. Remarkably, their measurements also favor higher ratios of $F_{\mathrm{LyC}} / F_{1500}$ than is expected of standard stellar population sythesis models. Both findings enhance the role of faint galaxies in driving reionization. 


\section{Modeling Reionization}

\subsection{Sources of Reionization}

Although it is widely believed that galaxies and quasars dominated reionization, they need not have acted alone. The theoretical search for plausible additional ionizing sources reads somewhat like a murder mystery in which the crime occurs in a crowded room. [40] recently argued that supernova remnants could have emitted up to $10 \%$ as many ionizing photons into the IGM as massive stars. The idea is that shocks produce a harder ionizing continuum than hot stars. Their more energetic photons experience a longer mean free path, hence their contribution to the photons that escape into the IGM could be significant.

Shocks from structure formation have also been forwarded [50, 16]. Here, the idea is that gas that accretes into relatively massive halos $\left(>3 \times 10^{10} \mathrm{M}_{\odot}\right.$ for [16] and $10^{11}-10^{12} \mathrm{M}_{\odot}$ for [50]) shocks as it accretes and virializes, converting gravitational potential energy into ionizing photons. [81] recently showed that, even if all baryons that fall into massive halos convert their gravitational potential energy into photons at the hydrogen ionization edge, they only produce 1 ionizing photon per three hydrogen atoms by $z=6$. This is far less than the likely contribution from galaxies. Nonetheless, these inquiries suggest that shocks may have impacted the IGM's thermal history, the topology of hydrogen reionization, and played a role in He II reionization.

Star formation in the progenitors of globular clusters (GCs) has also been proposed [61]. This is especially plausible if the total mass that formed in the progenitors of present-day GCs was $10 \times$ as large as their current stellar masses and if $f_{\text {esc }} \sim 1$. This idea has recently gained momentum from new observations of the metal abundances of GCs and the identification of candidate secondgeneration stars in the Milky Way's stellar halo. By using these observations to constrain the stellar initial mass function of GCs as well as the mass function of GCs themselves, [65] have shown that the contribution of GCs to reionization could have been quite large. If true, then the progenitors of massive GCs could have had masses of $10^{6} \mathrm{M}_{\odot}$ and will be visible to JWST.

More exotic sources of ionizing photons such as decaying dark matter particles and evaporating primordial black have not received much attention in recent years. We refer the reader to Section 3.4.4 of Furlanetto et al. [27] and Section 1 of Choudhury \& Ferrara [13] for an introduction to these alternatives.

\subsection{The Ionizing Escape Fraction from Galaxies}

The fraction of ionizing photons that escaped into the IGM, $f_{\text {esc }}$, is a crucial parameter that is regularly studied theoretically. [20] review both observational and theoretical inquiries into $f_{\text {esc }}$; interested readers may refer to their introduction for an overview. Broadly, analytical and numerical models predict values that span the full range from $0-1$. In order to understand this apparent lack of consensus, [20] use a simple model of stars embedded in a disk to explore how the properties of a galaxy's ISM and its stellar population modulate $f_{\text {esc }}$. They show that galaxies that deposit a large fraction of their ISM into a small number of high-density clumps possess a large $f_{\text {esc }}$. They also show that more intensely star-forming galaxies possess higher $f_{\text {esc }}$ partly because fewer baryons remain in the ISM and partly because the number of optically-thin sightlines through the ISM increases. They then attribute the wide range spanned by theoretical studies primarily to 
differences in the assumed gas masses and ISM topologies. The implication is that the predicted $f_{\text {esc }}$ will remain uncertain until the ISMs of high-redshift galaxies are understood.

\subsection{The IGM Recombination Rate}

In Section 3, I discussed efforts to measure the rate at which new ionizing photons were emitted into the IGM. Closing the loop requires us to know how many photons were required to achieve reionization, which in turn depends on the IGM's mean recombination rate. At early times $(z \geq 20)$, the Universe was relatively homogeneous and the recombination rate per volume in an ionized region was well-approximated by $\alpha\left\langle n_{\mathrm{H}}\right\rangle^{2}$, where $\alpha$ is the hydrogen recombination coefficient at a characteristic temperature such as $10^{4} \mathrm{~K}$ and $\left\langle n_{\mathrm{H}}\right\rangle$ is the mean hydrogen number density. By $z=6$, however, the IGM was inhomogeneous. Correcting for this involves multiplying the above rate by the clumping factor $C$, which depends on the probability distribution function of baryon densities, the topology of reionization - that is, which regions are ionized and which ones remain neutral — and the temperature of the ionized gas. [58] used realistic density fields culled from three-dimensional numerical simulations to predict that $C$ lies within the range $3-8$ by $z=6$. More recently, [70] used a different numerical model to predict that $C=2-4$ by $z=6$. Neither of these works included a self-consistent treatment of cosmological reionization, hence they were forced to make assumptions regarding the densities and temperatures of ionized regions. Their simulations also subtended different cosmological volumes at different spatial resolutions, hence it is possible that the slightly lower value of [70] owes to differences in resolution and the assumed topology of reionization. Broadly, however, both works prefer values $C$ that lie within the range of 3-10. This range is low enough to allow dwarf galaxies to drive reionization.

\subsection{One-Dimensional Models}

As we have seen, the history of reionization remains subject to many uncertainties such as the ionizing emissivity of stars and quasars, $f_{\mathrm{esc}}, C$, and the way in which these quantities evolve. A popular approach to understanding how these factors interact involves distilling reionization to a single equation that follows the growth of the ionized volume fraction with time. In this "photon-counting" exercise (for example, Equation 64 of [34]), the time rate of change of the neutral volume fraction is given by -(the ionizing emissivity per hydrogen atom from all sources) + (the recombination rate per hydrogen atom). The first term includes $f_{\mathrm{esc}}$ and the second includes C. [34] have shown that this formalism brings observations of the abundances of galaxies, quasars, IGM absorbers, and the optical depth to Thomson scattering (Section 2.3) into agreement with one another. Importantly, they assume a relatively low value $C=3$ at $z=6$, and they assume that the luminosity-weighted mean $f_{\text {esc }}$ increases at earlier times such that it exceeds $50 \%$ for $z>9.3$. These insights serve both as valuable inputs to complementary theoretical efforts and as predictions for future observational campaigns that will constrain $f_{\text {esc }}$.

\subsection{Three-dimensional Models}

Theorists have been attempting to model reionization in three dimensions for over a decade. A central goal is to model cosmic structure formation starting from $z \geq 100$ with as few physical assumptions as possible while reproducing as many observables as possible. I will divide the 
factors that models must consider into "agents", "processes", and "parameters". Agents include density fluctuations, Lyman limit systems, galaxies, and quasars. Processes include gas flows, the absorption of ionizing photons by an inhomogeneous IGM, recombinations, spectral hardening, and Jeans smoothing (Section 4.5.1). Emerging parameterizations that quantify these processes include the star formation efficiency as a function of dark matter halo mass; $f_{\text {esc }}$; the latent heat per IGM photoionization; and the IGM temperature, metallicity, ionization state, and recombination rate. To date, the chief observables have consisted of $\tau_{\mathrm{es}}$ (Section 2.3) and $x_{\mathrm{HI}}(z)$. In the near future, reionization models will begin confronting complementary observations of galaxies, the IGM metallicity, and the post-reionization Universe more generally.

The challenge of modeling these processes self-consistently is often summarized as follows: Properly sampling long-wavelength density fluctuations requires computational volumes to subtend at least $100 h^{-1} \mathrm{Mpc}$ [4]. Meanwhile, the lowest-mass dark matter halos that can form stars have a total mass of $\sim 10^{8} \mathrm{M}_{\odot}$; these must be resolved with $>100$ resolution elements. This implies a spatial dynamic range of $3 \times 10^{5}$. State-of-the-art hydrodynamic simulations currently achieve one tenth of this range. Folding in a treatment for radiation transport increases the computational expense by an additional 1-2 orders of magnitude. This dynamic range can be achieved within individual regions in "zoom-in" simulations, but not throughout a representative cosmological volume. In the face of these demands, progress is made by omitting or treating approximately some subset of the relevant physical processes or scales. Here I shall review recent efforts to this end. For a more comprehensive overview of cosmological radiation transport, see the code comparison papers [38, 39] or the review article by Trac \& Gnedin [78].

\subsubsection{Dark Matter + Radiation Transport}

Many studies use N-body (gravity-only) simulations to derive the cosmic density field, assume that gas follows matter, populate dark matter halos with ionizing sources following simple prescriptions, and then compute the inhomogeneous reionization history (see [78] for a summary of these works). The most recent of these calculations benefit from excellent spatial dynamic range, and they have simultaneously reproduced observations of $\tau_{\mathrm{es}}$ and $x_{\mathrm{HI}}$. The tradeoff is that they do not treat hydrodynamics and must therefore make assumptions regarding the scaling between ionizing luminosity and halo mass, the impact of photoionization heating on the IGM clumping factor, and the response of low-mass systems to photoionization heating. The latter processes are called "Jeans smoothing" and "Jeans suppression" and are simple to understand: When mildly overdense regions are photoheated, they expand until their self-gravity balances the local gas pressure (Jeans smoothing). Dark matter halos with total masses below $5 \times 10^{8} \mathrm{M}_{\odot}$ cannot accrete gas in such regions [24], hence their star formation is quenched within a few dynamical times (Jeans suppression).

Another approach is to derive the cosmological density field from precomputed hydrodynamic simulations, which can account (though not self-consistently) for Jeans smoothing by assuming a precomputed ionizing background. Such studies have also confirmed that galaxies could have driven reionization despite the Jeans suppression of low-mass sources. Interestingly, they fail to reproduce the post-reionization $x_{\mathrm{HI}}$ and ionization rate. This could owe to resolution limitations [23] or to observational systematics [2].

In a pathbreaking work, [77] combined a high-resolution N-body calculation of the density field with a medium-resolution hydrodynamical calculation that directly models Jeans smoothing 
while omitting Jeans suppression. They found that, following reionization, the IGM temperature and density are inversely related. This is because overdense regions are reionized and photoheated first, and have therefore had the most time to cool by $z=6$. Observational tests of predictions such as these will provide complementary constraints on the history of reionization.

\subsubsection{Semi-analytical Models}

Semi-analytical models (SAMs) [for example, 72] treat the formation of dark matter halos with relatively few approximations, then model how galaxies grow within those halos by using analytic prescriptions for baryonic processes such as gas infall, outflows, and star formation. By sidestepping the expense of discretizing the gas into parcels and integrating the gas equations, they sacrifice some realism in exchange for expanded flexibility. The governing physical parameters can, however, be tuned using observations and numerical simulations, improving their realism.

Benson et al. [6, and references therein] adapted a SAM to compute reionization self-consistently by modeling how ionizations and recombinations drive $x_{\mathrm{HI}}(z)$. Their approach accounts for Jeans suppression and includes a model for the clumping factor. It treats radiative processes such as selfshielding and shadowing only approximately and does not predict the topology of reionization. However, its ionizing emissivity can be tuned through observations of galaxy and quasar abundances in the post-reionization Universe, a major advantage. By varying parameters such as $f_{\text {esc }}$ and the strength of star formation feedback, they showed that galaxies could give rise to a range of reionization histories including some that are consistent with observations.

State-of-the-art SAMs use a statistical approach to confront many complementary observations simultaneously, yielding joint constraints on processes that regulate galaxy evolution [for example, 46]. Augmenting these models with treatments for inhomogeneous reionization will render them a powerful complement to numerical simulations.

\subsubsection{Semi-numerical Models}

If reionization was driven by galaxies, then the ionizing photons experienced a short mean free path and, on large enough scales, reionization was a local process. Semi-numerical models build upon this idea: if the number of ionizing photons produced within a region exceeds the number of hydrogen atoms (corrected for recombinations), then the region is probably ionized. These ideas were introduced within the context of an analytical model for reionization [25], and have since been adapted successfully for three-dimensional volumes [for example, 83]. They reproduce the spatial distribution of neutral and ionized regions predicted by the more exact numerical methods on scales larger than $\sim 1 h^{-1} \mathrm{Mpc}$ [84]. Given that they are inexpensive computationally, they can be combined with a statistical formalism to invert observations of the IGM ionization state and constrain the history of reionization [for example, 85]. They cannot treat processes that occur on spatial scales below which the assumption of local ionization does not apply such as spectral filtering, shadowing, and self-shielding within overdense regions.

\subsubsection{Cosmological Radiation-Hydrodynamic Simulations}

Simulations that treat dark matter, gas, and the radiation field self-consistently are the most realistic and computationally-intensive approach to modeling reionization. Their principal advantage is that they accurately model small-scale processes such as Jeans smoothing and Jeans suppression. 
The tradeoff is that their computational expense prevents them from modeling volumes that are larger than $\left(\sim 10 h^{-1} \mathrm{Mpc}\right)^{3}$, hence they do not capture long-wavelength density fluctuations.

Gnedin \& Fan [30] compared predictions from cosmological radiation hydrodynamic simulations with observations of the reionization-epoch Ly $\alpha$ forest and $\tau_{\mathrm{es}}$. They showed that highresolution simulations subtending 4 and $8 h^{-1}$ Mpc volumes yield converged predictions of the Ly $\alpha$ forest during the redshift range $5<z<6.2$ if the ionizing efficiency is adjusted to reproduce observations at $z=6 \pm 0.1$. The predicted $x_{\mathrm{HI}}(z>6.1)$ and $\tau_{\mathrm{es}}$ depend on the adopted mass resolution owing to star formation activity in systems whose mass lies near or below the resolution limit. The predicted IGM transmission grows less resolution-convergent in the reionization epoch because it is dominated by rare optically-thin regions. This work showed that simulations can reproduce the evolution of several observables of the reionization-epoch IGM. However, accounting for their sensitivity to resolution and volume limitations represents a formidable challenge.

In order to leverage the extensive observational constraints available from the post-reionization epoch, Finlator et al. [24] merged a well-studied galaxy evolution model with a radiation transport solver. They then used this framework to explore the impact of outflows and Jeans smoothing on star formation and reionization. They found that models combining strong outflows with an escape fraction of $f_{\text {esc }}=50 \%$ simultaneously reproduces the observed galaxy abundance at $z=6-8$ while completing reionization by $z=6$. They also studied how outflows and an ionizing background couple, finding that outflows promote star formation in dwarf galaxies by delaying reionization while suppressing it in more massive systems by coupling nonlinearly to the ionizing background (see also [59]). Their preferred model overproduced the ionizing background at $z<6$ while underproducing $\tau_{\text {es }}$. They interpreted this as evidence that $f_{\text {esc }}$ must evolve (consistent with [34]).

The problem of how to use radiation hydrodynamic simulations to improve our understanding of reionization-epoch galaxies and the IGM is a theoretical frontier owing to its computational expense. Over the next decade, efforts will be directed at two complementary goals. First, models will be generalized in order to expand the range of observables against which they can be tested. For example, artificial lines of sight through simulations will be compared with quasar absorption spectra in order to measure the reionization-epoch IGM's metallicity and temperature. Likewise, testing models against measurements of galaxies' colors and luminosities will constrain the abundance and activity in the putative ionizing population. These studies can be undertaken using existing simulations and will test current assumptions. Relaxing those assumptions is the second goal, and doing so requires expanding the dynamic range. Here there are two ways forward. The "brute-force" approach involves chipping away at the limitations of monolithic simulations through improved algorithms and hardware. The other approach uses high-resolution, small-volume simulations to tune the assumptions that underlie cosmological simulations. For example, [43] used small-volume simulations to estimate $C$ while other authors are using high-resolution simulations to model the star formation rates, metallicities, and $f_{\text {esc }}$ from reionization-epoch dwarf galaxies [11]. This approach will remain important until dynamic range limitations are overcome.

\section{Summary}

Over the past decade, $\mathrm{CMB}$ and galaxy observations have given rise to a consensus that galaxies could have driven hydrogen reionization, which in turn ended at some point before $z=6$. While 
little else is clear at the moment, a wide variety of studies are whittling away at the dominant questions. New observations of emission lines, the CMB, and redshifted 21 centimeter emission will soon measure the history of reionization. Ground- and space-based observations will locate and characterize the sources that caused it. On the theoretical side, "brute force" campaigns are expanding the dynamic range of numerical simulations. At the same time, approximate techniques are under development that will synthesize observational and theoretical insights into models that have broad applicability. All of this activity will render the coming decade a very active one, and there is every reason to believe that it will yield a much clearer picture of what went on during the second half of the first billion years.

\section{References}

[1] National Research Council. New Worlds, New Horizons in Astronomy and Astrophysics. Washington, DC: The National Academies Press, 2010.

[2] Aubert, D., \& Teyssier, R., Reionization Simulations Powered by Graphics Processing Units. I. On the Structure of the Ultraviolet Radiation Field, 2010, ApJ, 724, 244

[3] Barkana, R., \& Loeb, A., In the beginning: the first sources of light and the reionization of the universe, 2001, Phys. Rep., 349, 125

[4] Barkana, R., \& Loeb, A., Unusually Large Fluctuations in the Statistics of Galaxy Formation at High Redshift, 2004, ApJ, 609, 474

[5] Becker, R. H., Fan, X., White, R. L., et al., Evidence for Reionization at $z \sim 6$ : Detection of a Gunn-Peterson Trough in a $z=6.28$ Quasar, 2001, AJ, 122, 2850

[6] Benson, A. J., Sugiyama, N., Nusser, A., \& Lacey, C. G., The epoch of reionization, 2006, MNRAS, 369, 1055

[7] Bolton, J. S., Haehnelt, M. G., Warren, S. J., et al., How neutral is the intergalactic medium surrounding the redshift $z=7.085$ quasar ULAS J1120+0641?, 2011, MNRAS, 416, L70

[8] Bouwens, R. J., et al., Lower-Luminosity Galaxies could reionize the Universe: Very Steep Faint-End Slopes to the UV Luminosity Functions at $z \geq 5-8$ from the HUDF09 WFC3/IR Observations, 2011, arXiv:1105.2038

[9] Bouwens, R. J., Illingworth, G. D., Oesch, P. A., et al., UV-continuum slopes at $z \sim 4-7$ from the HUDF09+ERS+CANDELS observations: Discovery of a well-defined UV-color magnitude relationship for $z \geq 4$ star-forming galaxies, 2011, arXiv:1109.0994

[10] Bowman, J. D., \& Rogers, A. E. E., A lower limit of $\Delta z>0.06$ for the duration of the reionization epoch, 2010, Nature, 468, 796

[11] Bromm, V., \& Yoshida, N., The First Galaxies, 2011, ARA\&A, 49, 373

[12] Cen, R., \& Haiman, Z., Quasar Strömgren Spheres Before Cosmological Reionization, 2000, ApJ, 542, L75

[13] Choudhury, T. R., \& Ferrara, A., Physics of Cosmic Reionization, 2006, arXiv:astro$\mathrm{ph} / 0603149$

[14] Ciardi, B., \& Ferrara, A., The First Cosmic Structures and Their Effects, 2005, Space Science Reviews, 116, 625

[15] Dijkstra, M., Mesinger, A., \& Wyithe, J. S. B., The detectability of Ly $\alpha$ emission from galaxies during the epoch of reionization, 2011, MNRAS, 414, 2139 
[16] Dopita, M. A., Krauss, L. M., Sutherland, R. S., Kobayashi, C., \& Lineweaver, C. H., Reionizing the universe without stars, 2011, Ap\&SS, 335, 345

[17] Dunlop, J. S., McLure, R. J., Robertson, B. E., et al., A critical analysis of the ultraviolet continuum slopes $(\beta)$ of high-redshift galaxies: no evidence (yet) for extreme stellar populations at $z>6,2012$, MNRAS, 420, 901

[18] Fan, X., Narayanan, V. K., Strauss, M. A., et al., Evolution of the Ionizing Background and the Epoch of Reionization from the Spectra of $z \sim 6$ Quasars, 2002, AJ, 123, 1247

[19] Fan, X., et al., Constraining the Evolution of the Ionizing Background and the Epoch of Reionization with $z \sim 6$ Quasars. II. A Sample of 19 Quasars, 2006, AJ, 132, 117

[20] Fernandez, E. R., \& Shull, J. M., The Effect of Galactic Properties on the Escape Fraction of Ionizing Photons, 2011, ApJ, 731, 20

[21] Finkelstein, S. L., Papovich, C., Giavalisco, M., Reddy, N. A., Ferguson, H. C., Koekemoer, A. M., \& Dickinson, M., On the Stellar Populations and Evolution of Star-forming Galaxies at $6.3<z \leq 8.6,2010$, ApJ, 719, 1250

[22] Finkelstein, S. L., Papovich, C., Salmon, B., et al., CANDELS: The Evolution of Galaxy Rest-Frame Ultraviolet Colors from $z=8$ to 4, 2011, arXiv:1110.3785

[23] Finlator, K., Özel, F., \& Davé, R., A new moment method for continuum radiative transfer in cosmological reionization, 2009, MNRAS, 393, 1090

[24] Finlator, K., Davé, R., Özel, F., Galactic Outflows and Photoionization Heating in the Reionization Epoch, 2011, ApJ, 743, 169

[25] Furlanetto, S. R., Zaldarriaga, M., \& Hernquist, L., The Growth of H II Regions During Reionization, 2004, ApJ, 613, 1

[26] Furlanetto, S. R., Zaldarriaga, M., \& Hernquist, L., The effects of reionization on Ly $\alpha$ galaxy surveys, 2006, MNRAS, 365, 1012

[27] Furlanetto, S. R., Oh, S. P., \& Briggs, F. H., Cosmology at low frequencies: The $21 \mathrm{~cm}$ transition and the high-redshift Universe, 2006, Phys. Rep., 433, 181

[28] Glikman, E., Djorgovski, S. G., Stern, D., et al., The Faint End of the Quasar Luminosity Function at $z \sim 4$ : Implications for Ionization of the Intergalactic Medium and Cosmic Downsizing, 2011, ApJ, 728, L26

[29] Gnedin, N. Y., Cosmological Reionization by Stellar Sources, 2000, ApJ, 535, 530

[30] Gnedin, N. Y., \& Fan, X., Cosmic Reionization Redux, 2006, ApJ, 648, 1

[31] González, V., Labbé, I., Bouwens, R. J., Illingworth, G., Franx, M., \& Kriek, M., Evolution of Galaxy Stellar Mass Functions, Mass Densities, and Mass-to-light Ratios from $z \sim 7$ to $z \sim 4$, 2011, ApJ, 735, L34

[32] Grazian, A., et al., A critical analysis of the UV luminosity function at redshift $\sim 7$ from deep WFC3 data, 2011, A\&A, 532, A33

[33] Gunn, J. E., \& Peterson, B. A., On the Density of Neutral Hydrogen in Intergalactic Space., 1965, ApJ, 142, 1633

[34] Haardt, F., \& Madau, P., Radiative Transfer in a Clumpy Universe. IV. New Synthesis Models of the Cosmic UV/X-Ray Background, 2012, ApJ, 746, 125

[35] Henry, A. L., Martin, C. L., Dressler, A., Sawicki, M., \& McCarthy, P., The Faint-end Slope of the Redshift 5.7 Ly $\alpha$ Luminosity Function, 2012, ApJ, 744, 149 
[36] Hewett, P. C., Warren, S. J., Leggett, S. K., \& Hodgkin, S. T., The UKIRT Infrared Deep Sky Survey ZYJHK photometric system: passbands and synthetic colours, 2006, MNRAS, 367, 454

[37] Hu, E. M., Cowie, L. L., Barger, A. J., et al., An Atlas of $z=5.7$ and $z=6.5$ Ly $\alpha$ Emitters, 2010, ApJ, 725, 394

[38] Iliev, I. T., Ciardi, B., Alvarez, M. A., et al., Cosmological radiative transfer codes comparison project - I. The static density field tests, 2006, MNRAS, 371, 1057

[39] Iliev, I. T., Whalen, D., Mellema, G., et al., Cosmological radiative transfer comparison project - II. The radiation-hydrodynamic tests, 2009, MNRAS, 400, 1283

[40] Johnson, J. L., \& Khochfar, S., The Contribution of Supernovae to Cosmic Reionization, 2011, ApJ, 743, 126

[41] Kashikawa, N., et al., Completing the Census of Ly $\alpha$ Emitters at the Reionization Epoch, 2011, ApJ, 734, 119

[42] Kistler, M. D., Yüksel, H., Beacom, J. F., Hopkins, A. M., \& Wyithe, J. S. B., The Star Formation Rate in the Reionization Era as Indicated by Gamma-Ray Bursts, 2009, ApJ, 705, L104

[43] Kohler, K., Gnedin, N. Y., \& Hamilton, A. J. S., Large-Scale Simulations of Reionization, 2007, ApJ, 657, 15

[44] Komatsu, E., et al., Seven-year Wilkinson Microwave Anisotropy Probe (WMAP) Observations: Cosmological Interpretation, 2011, ApJS, 192, 18

[45] Loeb, A., \& Barkana, R., The Reionization of the Universe by the First Stars and Quasars, 2001, ARA\&A, 39, 19

[46] Lu, Y., Mo, H. J., Weinberg, M. D., \& Katz, N., A Bayesian approach to the semi-analytic model of galaxy formation: methodology, 2011, MNRAS, 416, 1949

[47] McGreer, I. D., Mesinger, A., \& Fan, X., The first (nearly) model-independent constraint on the neutral hydrogen fraction at $z \sim 6,2011$, MNRAS, 415, 3237

[48] McLure, R. J., Dunlop, J. S., de Ravel, L., et al., A robust sample of galaxies at redshifts $6.0<z<8.7$ : stellar populations, star formation rates and stellar masses, 2011, MNRAS, 418,2074

[49] McQuinn, M., Hernquist, L., Zaldarriaga, M., \& Dutta, S., Studying reionization with Ly $\alpha$ emitters, 2007, MNRAS, 381, 75

[50] Miniati, F., Ferrara, A., White, S. D. M., \& Bianchi, S., Ultraviolet background radiation from cosmic structure formation, 2004, MNRAS, 348, 964

[51] Morales, M. F., \& Wyithe, J. S. B., Reionization and Cosmology with 21-cm Fluctuations, 2010, ARA\&A, 48, 127

[52] Mortlock, D. J., Warren, S. J., Venemans, B. P., et al., A luminous quasar at a redshift of $z=7.085,2011$, Nature, 474,616

[53] Nestor, D. B., Shapley, A. E., Steidel, C. C., \& Siana, B., Narrowband Imaging of Escaping Lyman-continuum Emission in the SSA22 Field, 2011, ApJ, 736, 18

[54] Nilsson, K. K., \& Meisenheimer, K., Understanding Ly $\alpha$ emitters, 2009, New A Rev., 53, 37

[55] Oesch, P. A., Bouwens, R. J., Illingworth, G. D., et al., Expanded Search for $z \sim 10$ Galaxies from HUDF09, ERS, and CANDELS Data: Evidence for Accelerated Evolution at $z>8$ ?, 2012, ApJ, 745, 110 
[56] Ono, Y., Ouchi, M., Mobasher, B., et al., Spectroscopic Confirmation of Three z-dropout Galaxies at $z=6.844-7.213$ : Demographics of Ly $\alpha$ Emission in $z \sim 7$ Galaxies, 2012, ApJ, 744,83

[57] Ouchi, M., et al., Statistics of 207 Ly $\alpha$ Emitters at a Redshift Near 7: Constraints on Reionization and Galaxy Formation Models, 2010, ApJ, 723, 869

[58] Pawlik, A. H., Schaye, J., \& van Scherpenzeel, E., Keeping the Universe ionized: photoheating and the clumping factor of the high-redshift intergalactic medium, 2009, MNRAS, 394, 1812

[59] Pawlik, A. H., \& Schaye, J., Photoheating and supernova feedback amplify each other's effect on the cosmic star formation rate, 2009, MNRAS, 396, L46

[60] Pentericci, L., Fontana, A., Vanzella, E., et al., Spectroscopic Confirmation of $z \sim 7$ Lyman Break Galaxies: Probing the Earliest Galaxies and the Epoch of Reionization, 2011, ApJ, 743,132

[61] Ricotti, M., The Fate of the First Galaxies. I. Self-consistent Cosmological Simulations with Radiative Transfer, 2002, MNRAS, 336, L33

[62] Robertson, B. E., Ellis, R. S., Dunlop, J. S., McLure, R. J., \& Stark, D. P., Early star-forming galaxies and the reionization of the Universe, 2010, Nature, 468, 49

[63] Robertson, B. E., \& Ellis, R. S., Connecting the Gamma Ray Burst Rate and the Cosmic Star Formation History: Implications for Reionization and Galaxy Evolution, 2012, ApJ, 744, 95

[64] Schaerer, D., On the properties of massive Population III stars and metal-free stellar populations, 2002, A\&A, 382, 28

[65] Schaerer, D., \& Charbonnel, C., A new perspective on globular clusters, their initial mass function and their contribution to the stellar halo and the cosmic reionization, 2011, MNRAS, 413, 2297

[66] Schenker, M. A., Stark, D. P., Ellis, R. S., et al., Keck Spectroscopy of Faint $3<z<8$ Lyman Break Galaxies: Evidence for a Declining Fraction of Emission Line Sources in the Redshift Range $6<z<8,2012$, ApJ, 744, 179

[67] Shapiro, P. R., \& Giroux, M. L., Cosmological H II regions and the photoionization of the intergalactic medium, 1987, ApJ, 321, L107

[68] Shapley, A. E., Physical Properties of Galaxies from $z=2-4,2011$, ARA\&A, 49, 525

[69] Shull, J. M., France, K., Danforth, C. W., Smith, B., \& Tumlinson, J., HST/COS Observations of the Quasar HE 2347-4342: Probing the Epoch of He II Patchy Reionization at Redshifts $z=2.4-2.9,2010$, ApJ, 722, 1312

[70] Shull, M., Harness, A., Trenti, M., \& Smith, B., Critical Star-Formation Rates for Reionization: Full Reionization occurs at $z=7,2011$, arXiv:1108.3334

[71] Siana, B., Teplitz, H. I., Ferguson, H. C., et al., A Deep Hubble Space Telescope Search for Escaping Lyman Continuum Flux at $z \sim 1.3$ : Evidence for an Evolving Ionizing Emissivity, 2010, ApJ, 723, 241

[72] Somerville, R. S., Primack, J. R., \& Faber, S. M., The nature of high-redshift galaxies, 2001, MNRAS, 320, 504

[73] Spergel, D. N., Verde, L., Peiris, H. V., et al., First-Year Wilkinson Microwave Anisotropy Probe (WMAP) Observations: Determination of Cosmological Parameters, 2003, ApJS, 148, 175 
[74] Stark, D. P., Ellis, R. S., \& Ouchi, M., Keck Spectroscopy of Faint $3>z>7$ Lyman Break Galaxies: A High Fraction of Line Emitters at Redshift Six, 2011, ApJ, 728, L2

[75] Totani, T., Kawai, N., Kosugi, G., et al., Implications for Cosmic Reionization from the Optical Afterglow Spectrum of the Gamma-Ray Burst 050904 at $z=6.3,2006$, PASJ, 58, 485

[76] Tozzi, P., Madau, P., Meiksin, A., \& Rees, M. J., Radio Signatures of H I at High Redshift: Mapping the End of the "Dark Ages", 2000, ApJ, 528, 597

[77] Trac, H., Cen, R., \& Loeb, A., Imprint of Inhomogeneous Hydrogen Reionization on the Temperature Distribution of the Intergalactic Medium , 2008, ApJ, 689, L81

[78] Trac, H., \& Gnedin, N. Y., Computer Simulations of Cosmic Reionization, 2009, arXiv:0906.4348

[79] Willott, C. J., Delorme, P., Reylé, C., et al., The Canada-France High-z Quasar Survey: Nine New Quasars and the Luminosity Function at Redshift 6, 2010, AJ, 139, 906

[80] Wyithe, J. S. B., Hopkins, A. M., Kistler, M. D., Yüksel, H., \& Beacom, J. F., Determining the escape fraction of ionizing photons during reionization with the GRB-derived star formation rate, 2010, MNRAS, 401, 2561

[81] Wyithe, J. S. B., Mould, J., \& Loeb, A., The Shocking Truth: The Small Contribution to Hydrogen Reionization from Gravitational Infall, 2011, ApJ, 743, 173

[82] Yan, H., \& Windhorst, R. A., The Major Sources of the Cosmic Reionizing Background at $z \approx 6,2004, \mathrm{ApJ}, 600, \mathrm{~L} 1$

[83] Zahn, O., Zaldarriaga, M., Hernquist, L., \& McQuinn, M., The Influence of Nonuniform Reionization on the CMB, 2005, ApJ, 630, 657

[84] Zahn, O., Mesinger, A., McQuinn, M., et al., Comparison of reionization models: radiative transfer simulations and approximate, seminumeric models, 2011, MNRAS, 414, 727

[85] Zahn, O., Reichardt, C. L., Shaw, L., et al., Cosmic microwave background constraints on the duration and timing of reionization from the South Pole Telescope, 2011, arXiv:1111.6386 